\title{
COVID-19 Testing, Personal Protective Equipment, and Staffing Strategies Vary at Obstetrics Centers across the Country
}

\author{
Jasmine D. Johnson, $\mathrm{MD}^{1} \quad$ Emilie Melvin, $\mathrm{BS}^{2}$ \\ Brenna Hughes, $M^{5}$ Christina Shih-chi Han, MD $^{6}$ \\ ${ }^{1}$ Department of Obstetrics and Gynecology, University of North \\ Carolina School of Medicine, Chapel Hill, North Carolina \\ ${ }^{2}$ Georgetown University School of Medicine, Washington, District of \\ Columbia \\ ${ }^{3}$ Department of Obstetrics and Gynecology, Maternal and Child \\ Health Research Center, Perelman School of Medicine, University of \\ Pennsylvania, Philadelphia, Pennsylvania \\ ${ }^{4}$ Department of Obstetrics and Gynecology, University of South \\ Florida, Tampa, Florida \\ ${ }^{5}$ Department of Obstetrics and Gynecology, Duke University School \\ of Medicine, Durham, North Carolina \\ ${ }^{6}$ Department of Obstetrics and Gynecology, University of California at \\ Los Angeles, Los Angeles, California \\ 7 Department of Obstetrics, Gynecology and Reproductive Sciences, \\ University of California, San Francisco, San Francisco, California \\ ${ }^{8}$ Department of Obstetrics and Gynecology, Brown University Warren \\ Alpert Medical School, Providence, Rhode Island
}

Am J Perinatol 2020;37:1482-1484.

The continued safety and preservation of the health care workforce is vital during the ongoing coronavirus disease 2019 (COVID-19) pandemic. Observational data show that appropriate personal protective equipment (PPE)-including masks and eyewear are paramount to slowing the spread. ${ }^{1}$ Additionally, comprehensive screening strategies to help identify asymptomatic carriers will also protect the highest risk populations-including health care workers. ${ }^{2}$ A recent systematic review of 16 cohort studies estimated that asymptomatic carriers accounted for approximately 40 to $45 \%$ of positive severe acute respiratory syndrome coronavirus 2 (SARS-CoV-2) polymerase chain reaction tests, and this number is expected to rise as screening becomes more accessible. ${ }^{3}$ Sutton et al wrote in their commentary in the New England Journal of Medicine that the use of universal SARS-CoV-2 testing in all pregnant patients presenting for delivery revealed that most of the patients who were positive for SARS-CoV-2 at delivery were asymptomatic, and more than one in eight asymptomatic patients who were admitted to the labor and delivery unit were positive for SARS-CoV-2 during a period of increased community transmission. ${ }^{2}$ In an effort to characterize COVID-19 safety practices and resour-

received

September 10, 2020

accepted

September 11, 2020

published online

September 26, 2020
Address for correspondence Jasmine D. Johnson, MD, Division of Maternal-Fetal Medicine, University of North Carolina School of Medicine, 3010 Old Clinic Building, Campus Box 7516, Chapel Hill, NC 27599 (e-mail: jasmine.johnson@unchealth.unc.edu).

ces at academic and community hospital obstetric units across the United States, the Society for Maternal-Fetal Medicine (SMFM) administered a national survey to designated state liaisons regarding generalized testing and access to PPE in obstetrical units from April 7 to April 14, 2020. Results from the initial survey ("Survey I") found wide variation in universal testing policies and PPE use in obstetrical units across the United States. ${ }^{5}$ To determine how practices changed as rates across the country increased, SMFM re-administered the same survey from May 1, 2020 to May 22, 2020 ("Survey II").

\section{COVID-19-Positive Patients}

Similar to Survey I, this was a small but geographically diverse cohort. The survey was distributed electronically to 315 state liaisons. Of the 51 responses from 30 states and the District of Columbia, 65\% $(N=33)$ were from academic institutions with the remainder from community hospitals. There were 30 hospitals that responded to both the initial and repeat survey. With regard to COVID-19 cases during the pandemic, $45 \%(N=15 / 33)$ of those at academic institutions
Copyright $\odot 2020$ by Thieme Medical Publishers, Inc., 333 Seventh Avenue, New York, NY 10001, USA. Tel: +1(212) 760-0888.
License terms

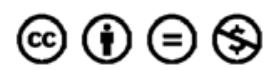




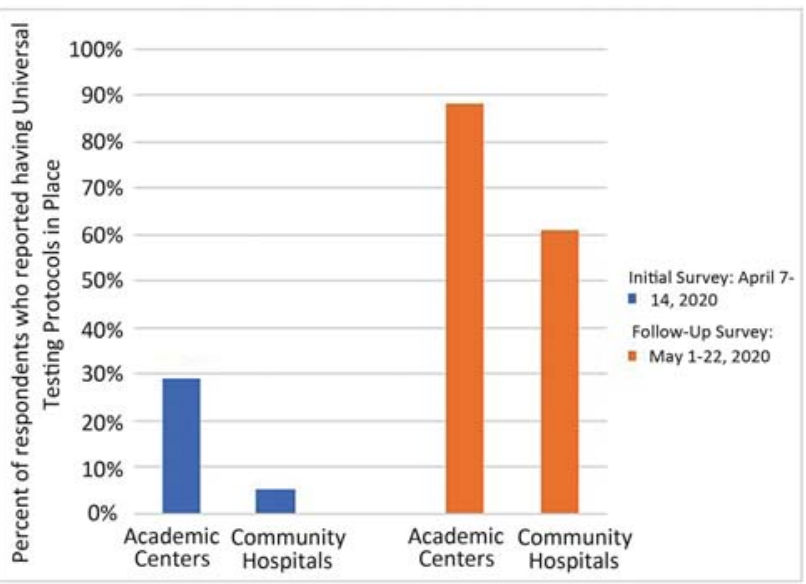

Fig. 1 Change in universal testing practices over time among community hospitals and academic centers.

reported $>20$ cumulative cases of COVID-19-positive pregnant patients at their institutions, and $11 \%$ (2/18) of those at community hospitals reported $>20$ cases of COVID-19-positive pregnant patients at their institutions. This represented a substantial increase in cumulative COVID-19-patient volume in less than 1 month; at the time of Survey I in April, 71\% of respondents had cared for fewer than three pregnant women with COVID-19.

\section{Universal Testing}

Among respondents to the Survey II, 78\% (40/51) reported that their hospitals were performing universal testing for women admitted for inpatient obstetrical care and 12\% (6/ 51 ) were considering but had not initiated this practice. This was a shift from Survey I, in which only $20 \%$ reported that their hospitals were performing universal testing. Only $10 \%$ $(5 / 51)$ of respondents reported no universal testing. As described previously, more academic hospitals than community hospitals reported universal testing, that is, $88 \%$ (29/33) versus $61 \%(11 / 18)(p=0.04)$ ( - Fig. 1). Finally, 82\% (14/17) of hospitals that admitted three or more COVID-19-positive patients per week were performing universal testing.

\section{Personal Protective Equipment}

Of the $94 \%$ of respondents who answered all questions about PPE, 75\% (36/48) of respondents reported using N95s for deliveries of COVID-19-positive patients or persons under investigation (PUIs), and 31\% (15/48) reported using N95s for all vaginal deliveries. Compared with Survey I findings, the use of N95 for delivery of all the patients rose slightly from $24 \%$ in Survey I to 31\% in Survey II. Universal N95 masking at the time of vaginal delivery was noted in $28 \%$ (9/32) of respondents at academic hospitals and 33\% (6/18) of respondents at community hospitals $(p=0.75)$.

\section{Staffing}

Out of the 50 respondents, 45 (90\%) indicated that their obstetrical unit has a surge plan in place and $20 \%$ of respondents $(10 / 50)$ reported using gynecologic or other staff to cover labor and delivery units. Of the 50 respondents, 32 had a protocol in place for pregnant health care workers. Most common examples of protocols included exemptions from treating COVID-19-positive patients or PUIs, work from home, or "off the front lines" after 37 weeks, and/or avoiding aerosol-generating procedures.

Survey II demonstrated that while the rate of COVID-19 is increasing among pregnant patients, there continues to be a lack of standardization in practices across the country. In particular, universal COVID-19 screening for obstetric admissions has become common at academic practices in a short amount of time but is still uncommon at community hospitals. This is likely due to a variety of factors including disparities between academic centers and community hospitals with regard to operating budgets, access to equipment, and would be an important area of additional research. Particularly, if PPE requirements vary by institution, universal testing should continue to be considered as a mainstay to protect our patients and our workforce.

Conflict of Interest

None declared.

\section{References}

1 Brooks JT, Butler JC, Redfield RR. Universal masking to prevent SARS-CoV-2 transmission-the time is now. JAMA 2020;324(07): 635-637

2 Sutton D, Fuchs K, D’Alton M, Goffman D. Universal screening for SARS-CoV-2 in women admitted for delivery. N Engl J Med 2020; 382(22):2163-2164

3 Oran DP, Topol EJ. Prevalence of asymptomatic SARS-CoV-2 infection: a narrative review. Ann Internal Med 2020;173(05):362-367

4 Coronavirus disease 2019 (COVID-19). Data on COVID-19 during pregnancy Centers for Disease Control and Prevention; 2020. Available at: https://www.cdc.gov/coronavirus/2019-ncov/casesupdates/special-populations/pregnancy-data-on-covid-19.html. Accessed July 22, 2020

5 Werner EF, Louis JM, Hughes B, Han CS, Norton ME, Srinivas SK. Community obstetrical units less likely than academic units to have universal COVID-19 testing. Am J Perinatol 2020;37(10): 1074-1076 